\title{
Differently phosphorylated forms of the cortactin homolog HS1 mediate distinct functions in natural killer cells
}

\author{
Boyd Butler, Diana H Kastendieck, and John A Cooper \\ Department of Cell Biology and Physiology, Washington University, Saint Louis, Missouri 63110, \\ USA
}

\begin{abstract}
Here we investigated the involvement of HS1, the hematopoietic cell-specific homolog of cortactin, in the actin-based functions of natural killer cells. Involvement of HS1 in T cell regulation has been established, as HS1 is required for the formation of immune synapses. 'Knockdown' of HS1 in natural killer cells resulted in defective lysis of target cells, cell adhesion, chemotaxis and actin assembly at the lytic synapse. Phosphorylation of the tyrosine residue at position 397 (Tyr397) was required for adhesion to the integrin ligand ICAM-1 and for cytolysis, whereas phosphorylation of Tyr378 was required for chemotaxis. Phosphorylation of Tyr397 was also required for integrin signaling and recruitment of integrins, adaptors and actin to the lytic synapse. Thus, HS1 is essential for signaling and actin assembly in natural killer cells, and the functions of the two phosphorylated tyrosine residues are distinct and separable.
\end{abstract}

An emerging frontier in cell and systems biology is the relationship between signaling networks and the cytoskeleton. Signaling pathways control the assembly and activity of the cytoskeleton, and in many cases, cytoskeletal elements control signaling pathways through positive and negative feedback. Here we show that the cortactin homolog HS1 (also called HCLS1 or LckBP1; A001149), noted before as being important for the formation of immune synapses ${ }^{1}$, has a critical function as an integrator between signaling pathways and actin cytoskeletal regulation.

The biology of natural killer (NK) cells in the innate immune system involves many receptormediated signaling and actin-assembly-based processes. Although much is known about these signaling and actin-assembly networks, relatively less is understood about how these two networks depend on and interact with each other. To address this issue, we studied HS1 as a candidate molecule for the transfer of information between the two networks.

NK cells are large, granular lymphocytes that recognize and kill transformed and virus-infected cells. NK cells 'decide' the fate of potential target cells according to the balance of activating and inhibitory signals that result from receptor-ligand interactions between NK cells and target cells $^{2}$. Most NK cells reside in the vasculature; thus, their cytolytic function begins with extravasation and chemotaxis toward target cells. These processes require integrin-mediated adhesion, signaling and actin assembly. When an NK cell encounters a potential target cell, NK receptors and integrins bind to ligands on the target cell surface; these interactions can lead to actin-mediated clustering of receptors, receptor-mediated signaling and the formation of a lytic synapse.

Correspondence should be addressed to B.B. (boyd.butler@wustl.edu).

Accession codes

UCSD-Nature Signaling Gateway (http://www.signaling-gateway.org): A002871, A001209 and A001149. 
By binding to its ligand ICAM-1 (A002871), the $\beta_{2}$ integrin LFA-1 (A001209) orchestrates NK cell-target cell interactions. LFA-1-deficient NK cells are defective in adhesion to target cells $^{3-5}$, and engagement of NK cell LFA-1 alone is sufficient for adhesion, lytic synapse formation and polarization of cytolytic granules toward the target cell ${ }^{6}$. LFA- 1 on hematopoietic cells must be activated to achieve a high-affinity state ${ }^{7}$. 'Inside-out' activation of the GTPase Rap1 by chemokines induces integrin-mediated adhesion and migration ${ }^{8,9}$, and Rap1-deficient cells show impaired LFA-1-mediated adhesion ${ }^{10}$. The adaptor cytohesin-1 binds to the cytoplasmic domain of $\beta_{2}$ integrin and promotes LFA-1 activation ${ }^{11}$. Activating NK cell receptors such as NKG2D recruit the adaptor molecule DAP10, which becomes tyrosine-phosphorylated and promotes the association of specific signaling and scaffolding molecules, including Grb2, Vav1 (a member of the Dbl family of guanine nucleotide-exchange factors in hematopoietic cells) and phosphatidylinositol-3-OH kinase $(\mathrm{PI}(3) \mathrm{K})^{12}$. These signaling proteins promote the formation and stabilization of the lytic synapse and activation of the cytolytic response $\mathrm{e}^{2,13,14}$.

The actin cytoskeleton is critical in NK cell function and is remodeled extensively during interactions of NK cells with target cells ${ }^{15}$. Inhibition of actin assembly by cytochalasin D prevents the formation of a stable competent lytic synapse ${ }^{16}$. In motile cells, polymerization of a branched network of actin filaments pushes the cell membrane forward. The Arp2/3 complex, which nucleates actin filament assembly from the sides of existing 'mother' actin filaments thereby forming a branched network, can be activated by the WASp, cortactin and WAVE/Scar families of actin regulators. WASp and other actin regulators localize to the lytic synapse $^{17}$, and NK cells from patients lacking WASp show impaired polarization, less actin assembly at the lytic synapse and impaired cytolytic function ${ }^{18}$. Cortactin, identified as a substrate of the tyrosine kinase $\mathrm{Src}^{19}$, binds $\mathrm{Arp} 2 / 3$ and actin filaments and thereby promotes formation and stabilization of the branched filament network ${ }^{20}$. HS1 is the hematopoietic cellspecific homolog of cortactin ${ }^{21}$. Like cortactin, HS1 is a substrate of Src family tyrosine kinases, which are activated after activation of B cells and T cells $1,21-24$. HS1-deficient mice have defects in antigen-induced proliferation of B cells and T cells 25,26 , and HS1 is important for the formation of immune synapses in T cells ${ }^{1}$. In platelets, HS1 is required for collagen receptor signaling, aggregation and thrombus formation 27 .

The function of HS1 during the formation of lytic synapse by NK cells has not been investigated, and the function of tyrosine phosphorylation of HS1 is not well understood in any system. Here we found that knockdown of HS1 in NK cells impaired the formation of lytic synapses and cytolytic activity. In addition, we found that the tyrosine residue at position 397 (Tyr397) of HS1 was required for adhesion to ICAM-1, lytic synapse formation and cytolytic activity. In contrast, HS1 Tyr378 was dispensable for those processes but was necessary for chemotaxis by a mechanism independent of Tyr397.

\section{RESULTS}

\section{HS1 localization and phosphorylation}

To investigate the involvement of HS1 in various NK cell functions, we first examined the localization and phosphorylation status of HS1 during the formation of lytic synapses. We incubated primary NK cells or NKL cells (NK cells derived from a human NK cell leukemia) expressing green fluorescent protein (GFP)-tagged HS1 (GFP-HS1) together with K562 target cells (human myelogenous leukemia cells). Within 5 min, GFP-HS1 localized to the cell-cell contact site (Fig. 1a and Supplementary Movies 1 and 2 online). To test a defined synthetic target in place of the K562 cells, we used Dynabeads coated with the NK receptor ligand UL16binding protein (ULBP) and the LFA-1 ligand ICAM-1. In primary NK cells, GFP-HS1 quickly localized to the cell-bead interface (Fig. 1b). In both experiments, HS1 became tyrosinephosphorylated with a similarly rapid time course (Fig. 1). To further investigate the 
involvement of HS1 in NK cell function, we inhibited HS1 expression by tetracycline-inducible expression of HS1-specific short hairpin RNA (shRNA). Two different HS1 targeting constructs (HS1A and HS1B) suppressed HS1 protein expression equally within $72 \mathrm{~h}$ of the addition of doxycycline (Fig. 2a). A nontargeting version of HS1A had no effect on HS1 expression. In primary NK cells, the degree of HS1 suppression by HS1A and HS1B was partial, not complete, even at $72 \mathrm{~h}$. After $72 \mathrm{~h}$ of exposure to doxycycline, we transfected primary NK cells and NKL cells by nucleofection with GFP-tagged wild-type and point mutant versions of HS1. After $36 \mathrm{~h}$, cells had higher expression of the exogenous HS1 constructs than of endogenous HS1 but not by more than two- to threefold (Fig. 2b). Tyr378 and Tyr397 of HS1 are phosphorylated in vivo ${ }^{1}$. To test the functional importance of tyrosine phosphorylation, we constructed single and double point mutants of HS1, replacing the tyrosine residues with phenylalanine (Y378F, Y397F and Y378F,Y397F). The GFP-tagged Y378F and Y397F HS1 mutants were phosphorylated to a lesser extent than was the wild-type protein, and the Y378F, Y397F HS1 mutant showed very little tyrosine phosphorylation (Fig. 2b). Therefore, we established that HS1 is rapidly tyrosine-phosphorylated after adhesion to targets and that we were able to inducibly 'knock down' HS1 and re-express wild-type or mutant HS1 constructs.

\section{Function of HS1 in NK cell-mediated cytolysis}

To test the function of HS1 in cytolysis, we measured the capacity of NK cells with HS1 'knocked down' (HS1-knockdown cells) to lyse K562 target cells. Primary NK cells and two NK cell lines (NKL and NK92) with less expression of HS1 had much less cytolytic activity (Fig. 2c). Cytotoxicity was normal in cells expressing nontargeting shRNA (data not shown). Expression of shRNA-resistant wild-type HS1, but not GFP alone, restored the cytolytic phenotype of HS1-knockdown cells (data not shown).

To determine if tyrosine phosphorylation of HS1 is important for NK cell cytotoxicity, we attempted to 'rescue' HS1-knockdown cells with HS1 point mutants. HS1-knockdown NK cells transfected by nucleofection with the Y397F or Y378F, Y397F HS1 mutant showed little to no restoration of cytotoxicity (Fig. 2c). In contrast, the Y378F HS1 mutant produced partial restoration. As the wild-type and mutant HS1 constructs were tagged with GFP, we were able to confirm that low expression did not cause the failure to restore cytotoxicity (Fig. 2b). Therefore, HS1 is important in NK cell cytotoxicity, and tyrosine phosphorylation of HS1 seems to be important for this process. To test for dominant negative or overexpression effects, we expressed wild-type HS1 or the Y378F, Y397F HS1 mutant in control primary NK cells with endogenous HS1 expression and found no effect on cytolytic activity (Supplementary Fig. 1 online).

\section{HS1 in integrin-mediated adhesion}

NK cells reside mainly in the peripheral circulation and undergo extravasation before interacting with potential target cells. Firm adhesion of the NK cells to endothelial cells and the extracellular matrix is mediated by surface-expressed integrins, which are maintained in an inactive state 28,29 . We therefore investigated the function of HS1 in integrin-mediated adhesion. NK cells express LFA-1 $\left(\alpha_{\mathrm{L}} \beta_{2}\right)$, which binds to ICAM-1 expressed on potential target cells. NK cells also express the integrin VLA- $4\left(\alpha_{4} \beta_{1}\right)$, which interacts with fibronectin in the extracellular matrix. We found that HS1 became tyrosine-phosphorylated when wild-type primary NK cells were allowed to adhere to ICAM-1; in contrast, only minimal HS1 phosphorylation was induced by adhesion to fibronectin (Fig. 3a). Expression of wild-type HS1 or Y378F HS1 in HS1-knockdown cells restored tyrosine phosphorylation of HS1 on ICAM-1 but expression of Y397F or Y378F, Y397F HS1 did not (Fig. 3a). Thus, Tyr397 is the main tyrosine residue phosphorylated after the adhesion of NK cells to ICAM-1. 
We next assessed the ability of NK cells to adhere to ICAM-1 or fibronectin by plate assays and time-lapse movies. Control NK cells activated by phorbol 12-myristate 13-acetate (PMA) adhered well to either protein (Fig. 3b and Supplementary Movies 3-6 online). PMA was required for adhesion (data not shown). HS1-deficient primary NK cells showed impaired adhesion to ICAM-1 but normal adhesion to fibronectin (Supplementary Movies 7-9 online). Wild-type HS1 or Y378F HS1, but not Y397F or Y378F, Y397F HS1, restored the adhesion of HS1-knockdown NK cells to ICAM-1 (Supplementary Movies 10-13 online). Thus, Tyr397 but not Tyr378 is important for adhesion to ICAM-1. To test for dominant negative or overexpression effects, we expressed wild-type HS1 or the Y378F, Y397F HS1 mutant in NK cells in which endogenous HS1 was not 'knocked down'. We found no effect on adhesion to ICAM-1 (Supplementary Fig. 1).

To assess the state of the actin cytoskeleton during adhesion, we allowed primary NK cells to adhere to ICAM-1 or fibronectin for $15 \mathrm{~min}$ in the presence of PMA and stained the cells with fluorescent phalloidin. At the cell-substrate contact site, total internal reflection fluorescence (TIRF) microscopy showed that control primary NK cells on ICAM-1 had actin-rich filopodial structures, a cortical ring of actin and punctate central actin staining (Fig. 3c). Loss of HS1 resulted in diffuse actin staining with few or no filopodial structures and no cortical accumulation of actin. Expression of wild-type HS1 or Y378F HS1 restored the wild-type actin phenotype but expression of Y397F or Y378F, Y397F HS1 did not. On fibronectin, primary NK cells spread well and had many actin-rich structures, similar to those seen on ICAM-1, and HS1 knockdown had no observable effects on the actin cytoskeleton. To investigate the mechanism by which HS1 influences actin assembly, we determined the localization of HS1 and other proteins known to regulate actin dynamics. In control cells adhering to ICAM-1, GFP-HS1 was localized to filopodial structures and the cortical ring, with central punctate staining, as shown above for actin (Fig. 3d). Double staining with fluorescent phalloidin confirmed that GFP-HS1 localized together with actin. Vav1, $\beta_{2}$ integrin and WASp also localized together with GFP-HS1. In contrast, NK cells adhering to fibronectin showed diffuse GFP-HS1 localization. In these cells, actin, $\beta_{2}$ integrin, Vav1 and WASp were localized to pseudopodial extensions at the edge of the cell-surface contact site and in coarse structures in the center of the cell (Fig. 3d). Therefore, we established that phosphorylation of Tyr397 was required for adhesion to ICAM-1 and that after adhesion to ICAM-1, HS1 localized together with the actin cytoskeleton as well as key proteins involved in actin cytoskeletal dynamics.

\section{Function of HS1 in integrin activation}

Integrins constitutively expressed on the surface of NK cells are maintained in an inactive state to prevent unwarranted adhesion and must be activated to achieve a high-affinity state. Integrinmediated attachment of NK cells to target cells provides an early signal in the cytolytic response, and downregulation of the surface expression of integrins disrupts NK cell-mediated killing 4,30 . Therefore, we sought to determine whether the lack of firm adhesion to ICAM-1 by HS1-deficient NK cells was due to an inability of LFA-1 to achieve a high-affinity state. We assayed the high-affinity state of LFA- 1 by staining with the antibody mAB24, as mAB24 recognizes only the active conformation of LFA-1. We incubated primary NK cells with the chemokine SDF-1a, which triggers 'inside-out' signaling and promotes greater integrin affinity ${ }^{7}$. Loss of HS1 inhibited the conversion of LFA-1 from a low-affinity state to a highaffinity state (Table 1). The diminished staining of HS1-deficient NK cells with mAb24 was not due to lack of LFA-1 surface expression (data not shown). Expression of wild-type HS1 or the Y378F HS1 restored mAb24 staining but expression of Y397F or Y378F,Y397F HS1 did not. In contrast, loss of HS1 did not affect the conversion of VLA-4, the integrin used in the adhesion of NK cells to fibronectin, to the high-affinity state, as determined by staining with the monoclonal antibody 9EG7, specific for integrin $\beta_{1}$ (Table 1). In addition, we assessed the function of HS1 in the 'outside-in' activation of LFA-1. We incubated NKL cells with 
$\mathrm{Mn}^{2+}$ to promote a high-affinity state of surface-expressed LFA-1. Loss of HS1 had no effect on the $\mathrm{Mn}^{2+}$-induced conversion to high-affinity LFA-1, which suggested that HS1 regulates 'inside-out' rather than 'outside-in' activation of LFA-1.

Next, we investigated how HS1 influences signaling pathways 'downstream' of integrin engagement. HS1-knockdown primary NK cells on ICAM-1 showed less phosphorylation of the Src-family kinase Lyn, Pyk2 and Vav1 but normal phosphorylation of the tyrosine kinase Syk and PI(3)K (Fig. 4a). In contrast, loss of HS1 did not disrupt integrin-based signaling after adhesion to fibronectin. We also assessed the binding of signaling proteins to LFA-1. Vav1 immunoprecipitated with LFA-1 in control NK cells, and HS1 knockdown resulted in a complete loss of this association (Fig. 4b). Expression of wild-type HS1 or Y378F HS1 restored the association of Vav1 with LFA-1 in HS1-knockdown cells but expression of Y397F or Y378F, Y397F HS1 did not. In addition, we found that HS1 knockdown disrupted the association of cytohesin-1, which is critical for LFA-1 activation ${ }^{11}$, with LFA-1. Expression of wild-type HS1 or Y378F HS1 restored the association of cytohesin-1 with LFA-1 in HS1knockdown cells but expression of Y397F or Y378F,Y397F HS1 did not. Finally, the association of Pyk2 with LFA-1 was diminished slightly by the loss of HS1 expression but not to the extent noted for Vav1 and cytohesin-1 (Fig. 4b). Rho-family GTPases are critical for the actin assembly needed for integrin-mediated adhesion $31-33$. We examined the function of HS1 in the activation of Rho-family GTPases in NK cells on ICAM-1, using 'CRIB' precipitation assays to measure the active forms of the Rho-family GTPases Rac1, Cdc42 and Rap1. The conserved 'CRIB' (Cdc42- and Rac-interactive binding) motif is essential for protein-protein interactions with the active GTP-bound state of Rho family GTPases. In control primary NK cells on ICAM-1, we found activation of Rac, Cdc42 and Rap1 at 5 min that reached a plateau at $10 \mathrm{~min}$ after adhesion (Fig. 4c). Loss of HS1 resulted in diminished activation of all three proteins, and their activation was restored by the expression of wild-type HS1 or Y378F HS1 but not by expression of Y397F or Y378F, Y397F HS1.

Because HS1 localized to the lytic synapse and knockdown of HS1 impaired LFA-1 activation and signaling, we investigated the protein composition and architecture of the lyticsynapse. In control NKL cells stably expressing GFP-labeled proteins, we found that actin, LFA-1, Vav1 and WASp localized to the lytic synapse (Fig. 5a). However, in HS1-knockdown NKL cells, recruitment of actin, LFA-1, Vav1 and WASp to the lytic synapse was impaired. We evaluated the time course of the localization of actin and Vav1 to the lytic synapse and found that each was impaired at all time points examined (Fig. 5b). Wild-type HS1 or Y378F HS1 but not Y397 or Y378F, Y397F HS1 'rescued' these defects (Fig. 5c). The recruitment of HS1 itself to the lytic synapse required Tyr397, in that expression of wild-type HS1 or Y378F HS1 restored HS1 localization, whereas expression of the Y397 or Y378F,Y397F HS1 did not. Therefore, we established that phosphorylation of HS1 Tyr397 was required for LFA-1 activation and LFA-1 mediated signaling that contributes to proper formation of lytic synapses.

\section{HS1 and chemotaxis}

Because Y378F HS1 facilitated cytolysis to an extent intermediate between that of wild-type HS1 and the Y397F HS1 mutant (Fig. 2c), we investigated further the function of HS1 Tyr378. Initially, cytolysis assays over a time course in 96 -well flat-bottomed plates yielded a similar intermediate phenotype for Y378F (Fig. 6a). The rate of cytolysis mediated by Y378F HS1 was lower, similar to that mediated by Y397F or Y378F, Y397F HS1, until approximately 2.5 $\mathrm{h}$, after which the rate of cytolysis with Y378F HS1 increased, which suggested that NK cells expressing the Y378F HS1 mutant might be delayed in reaching potential target cells. To test that hypothesis, we first modified the assay to eliminate the need for cells to migrate to their targets by using 96 -well round-bottomed plates instead of 96-well flat-bottomed plates. In this setting, although HS1 knockdown resulted in a loss of cytolytic activity, we noted full 'rescue' 
with Y378F HS1 relative to wild-type HS1 but no 'rescue' with Y397F or Y378F, Y397F HS1 (Fig. 6b). Next, we challenged NK cells to migrate further to locate target cells by doing the assay in six-well flat-bottomed plates. In this setting, as expected, cytolysis occurred at a much slower rate and loss of HS1 impaired cytolysis. However, the defect was 'rescued' only by expression of wild-type HS1; Y378F, Y397F and Y378F, Y397F HS1 failed to provide even partial 'rescue' (Fig. 6c). Next, we determined whether HS1 Tyr378 was phosphorylated during chemotaxis. In NK cells adhering to fibronectin, HS1 tyrosine residues were only minimally phosphorylated. In contrast, treatment with SDF-1a induced robust phosphorylation of wildtype HS1 and Y397F HS1 but only minimal phosphorylation of Y387F or Y378F,397F HS1 (Fig. 7a). We concluded that Tyr378 but not Tyr397 is phosphorylated after induction of chemotaxis signaling.

To investigate cell migration further, we did assays of 'haptotaxis' (movement induced by an adhesion gradient) and chemotaxis with Transwell plates. To assess haptotaxis, we placed cells in the upper chamber on a Transwell membrane coated with fibronectin and allowed them to migrate toward the lower membrane surface, which had been coated with a higher concentration of fibronectin. In the absence of a chemoattractant, HS1-deficient NK cells migrated to the lower membrane surface to the same degree as did control NK cells (Fig. 7b). To assess chemotaxis, we added SDF-1a to the lower chamber and coated the top surface of the Transwell membrane with fibronectin. In this assay, HS1-deficient NK cells migrated poorly relative to control cells (Fig. 7b). Expression of wild-type HS1 'rescued' the migration defect, as did Y397F HS1, but expression of Y378F or Y378F, Y397F HS1 did not. When we used ICAM-1 to coat the membrane in these assays, cell migration was impaired by the loss of HS1, and this defect was 'rescued' by expression of wild-type HS1 but not any of the HS1 tyrosine mutants (data not shown). These results are consistent with the failure of the Y397F HS1 mutant to restore adhesion to ICAM-1 (Fig. 3b,c).

Although integrin-mediated adhesion is thought to be responsible for maintaining an adhesive state during leukocyte chemotaxis, evidence has shown that chemokine-induced leukocyte motility can occur in the absence of stable integrin-mediated adhesion ${ }^{34}$. To determine if primary NK cells required integrin-mediated adhesion during Transwell migration, we treated NK cells with antibodies blocking LFA- $1\left(\beta_{2}\right)$ or VLA-4 $\left(\beta_{1}\right)$ and noted that blocking $\beta_{2}$ integrin disrupted both chemotactic and haptotactic migration on fibronectin but blocking $\beta_{1}$ integrin did not (Supplementary Fig. 2 online).

To examine cell migration directly, we imaged live NK cells on fibronectin in the presence of SDF-1a. Here we added SDF-1a in the form of a pellet of hardened agarose to create a chemoattractant gradient and thereby orient cell migration. Control NK cells moved en masse in the direction of SDF-1a (Supplementary Movie 14 online), whereas HS1-deficient NK cells did not (Supplementary Movie 15 online). Directional migration was restored in HS1knockdown cells by expression of Y397F HS1 but not by expression of Y378F HS1 (Supplementary Movies 16 and 17 online). At higher magnification, it was apparent that control NK cells sent out protrusions in the direction of migration (Supplementary Movie 18 online), whereas HS1-knockdown cells had substantial randomly oriented protrusions and movement (Supplementary Movie 19 online). Directional migration was restored in HS1-knockdown cells by expression of Y397F HS1 but not by expression of Y378F HS1 (Supplementary Movies 20 and 21 online). These results collectively indicate that Tyr378 is specifically required for chemotaxis.

Ligation by SDF-1a of its G protein-coupled receptor CXCR4 results in the activation of many signaling pathways, including the phosphorylation and activation of phospholipase C-b and the kinase PAK1 (refs. 35,36), which induce actin reorganization through Rac1 and Cdc42 (refs. 37,38 ). We sought to determine whether HS1 regulated these chemoattractant-induced 
signaling pathways. We found that loss of HS1 in NK cells had little effect on the phosphorylation of phospholipase $\mathrm{C}-\mathrm{b}$; however, phosphorylation of PAK1, PI(3)K and Vav1 was inhibited (Fig. 7c). Expression of wild-type HS1 fully restored the phosphorylation of Vav1 and PI(3)K but expression of Y378F or Y378F, Y397F did not. Expression of Y397F HS1 completely restored PI(3)K activation and partially restored Vav1 activation. We then measured the activation of RhoA, Rac1 and Cdc42. In control NK cells, RhoA activation decreased after the addition of SDF-1a in a way independent of HS1 expression (Fig. 7d). In contrast, Rac 1 and Cdc 42 activation were induced within 5 min of SDF-1a treatment, plateaued at $10 \mathrm{~min}$ and persisted for at least $30 \mathrm{~min}$. This activation of Rac 1 and $\mathrm{Cdc} 42$ was diminished by knockdown of HS1 and was restored by expression of wild-type HS1 or Y397F HS1 but not by expression of Y378F or Y378F,Y397F HS1. Thus, Tyr378 is important for the transmission of early signals 'downstream' of SDF-1a to Vav1, Rac1 and Cdc42.

\section{HS1 and NK receptor signaling}

Because NK receptor ligation strongly influences the activation status of NK cells, we investigated whether HS1 was important for signaling 'downstream' of NK receptors. We first determined whether localization of the NKG2D-binding protein DAP10 to the lytic synapse depended on HS1. In primary NK cells incubated for 10 min with K562 cells, we noted that DAP10 did localize to the lytic synapse despite knockdown of HS1 (Fig. 8a). To isolate the effects of the individual receptors, we substituted Dynabeads coated with ULBP and ICAM-1 for the K562 target cells. DAP10 was rapidly phosphorylated after exposure to the coated beads in a way unaffected by knockdown of HS1 (Fig. 8b). Phosphorylation of Lyn, PI(3)K and Vav1 also occurred after NKG2D ligation and was somewhat lower in HS1-knockdown cells. Phosphorylation of PI (3) K and Vav1 was restored by expression of wild-type HS1 or Y378F HS1 but not by expression of Y397F or Y378F, Y397F HS1. After NKG2D recruits the adaptor DAP10, phosphorylated DAP10 provides docking sites for other molecules, including PI(3) $\mathrm{K}, \mathrm{Grb} 2$ and Vav1. We sought to determine whether loss of HS1 impaired the recruitment of these molecules to phosphorylated DAP10. In primary NK cells incubated with Dynabeads coated with ULBP and ICAM-1, the association of Grb2 and PI(3)K with DAP10 was somewhat lower, whereas the association of Vav1 with DAP10 was severely impaired after HS1 knockdown (Fig. 8c). Expression of wild-type HS1 or Y378F HS1 restored the association of Vav1 with DAP10 but expression of Y397F or Y378F, Y397F HS1 did not. NKG2D-induced production of interferon-g and tumor necrosis factor was also lower after knockdown of HS1 and was restored by expression of wild-type HS1 or Y378F HS1 but not by expression of Y397F or Y378F, Y397F HS1 (Fig. 8d). Finally, we found that activation of Rho-family GTPases triggered by NKG2D stimulation depended on HS1. Knockdown of HS1 diminished NKG2D-induced phosphorylation of Rac1 and Cdc42, and this phosphorylation was restored by expression of wild-type HS1 or Y378F HS1 (Fig. 8e). Expression of Y397F or Y378F,Y397F HS1 restored the activation of Rac1 and Cdc42 but with delayed kinetics and without maintenance beyond $15 \mathrm{~min}$. Analysis of Rap1 activation, required for adhesion to ICAM-1, produced similar results. Rap1 was activated fully within 5 min and activation was maintained for at least $15 \mathrm{~min}$. Loss of HS1 diminished Rap1 activation, which was restored by expression of wild-type HS1 or Y378F HS1 but not by expression of Y397F or Y378F, Y397F HS1. Thus, we determined that phosphorylation of HS1 Tyr397 was required for the transmission of HS1-independent early NK receptor signaling to 'downstream' signaling molecules such as Vav1 and their subsequent association with immediate 'upstream' molecules such as DAP10.

\section{DISCUSSION}

The biology of NK cells includes many processes that involve receptor-mediated signaling and actin assembly. Here we have demonstrated involvement of HS1, a protein uniquely poised at 
the interface between signaling and actin assembly, in transferring information between these two networks. First, we found that HS1 was necessary for many of the diverse processes essential for NK cell function, including adhesion, integrin activation, chemotaxis, lytic synapse assembly and cytolysis. Tyrosine phosphorylation of HS1 was necessary in every case, but we found that the two tyrosine residues of HS1 had distinct and separable functions. Second, we found that the initiation and/or maintenance of many of the receptor-based signaling pathways in NK cells, including those emanating from integrins and NK receptors, depend on one HS1 tyrosine residue. The integrin signaling function of HS1 was specific for LFA-1, the integrin required for the adhesion of NK cells to target cells, and only the most immediate signaling from NK receptors and integrins was independent of HS1. Therefore, HS1 occupies a central position in generating and maintaining signals, as well as directing actin assembly. Other pathways can promote or maintain actin assembly, which may reinforce or enhance the action of HS1, providing feedback to generate and maintain the signals 'downstream' of HS1.

One of our main goals was to determine the functional importance of tyrosine phosphorylation of HS1 in NK cells. Tyrosine phosphorylation is necessary for the function of cortactin in certain cell systems ${ }^{39,40}$, and tyrosine phosphorylation of HS1 at Tyr378 and Try397 has been suggested to be important for activation induced by immune receptors, including B cell and $\mathrm{T}$ cell receptors and FcgRIII, and for platelet activation 1,24-28. Here we found that tyrosine phosphorylation of HS1 was also necessary for all of the functions of HS1 in NK cells. More notably, we found evidence that the two tyrosine residues of HS1 had distinct and separable functions. Tyr397 but not Tyr378 was required for NK cell-target cell synapse formation and cytolysis, as well as for NK receptor signaling. In addition, Tyr397 was specifically required for adhesion, cell spreading and nonchemotactic migration in response to LFA-1-based signals but not for the same processes mediated by VLA-4. In contrast, Tyr378 but not Tyr397 was necessary for NK cell chemotaxis.

We found that HS1 was necessary for NK cell signaling 'downstream' of immediate membrane-proximal events. Integrin ligation and activation initiates signaling that promotes actin cytoskeletal reorganization in NK cells ${ }^{4,5,16,17}$. In terms of signaling from LFA-1, phosphorylation of Syk was unaffected by the loss of HS1, but activation of Lyn, Pyk2, Vav1, $\mathrm{PI}(3) \mathrm{K}$ and Rho-family proteins was impaired. As for the activation of NK receptors, recruitment of the adaptor DAP10 did not depend on HS1 but the activation and recruitment of components 'downstream' of DAP10 did. In all these cases, the Y397 HS1 mutant but not the Y378 HS1 mutant was crucial for HS1 function.

HS1 is necessary for integrin activation. LFA-1 on the surfaces of circulating NK cells is maintained in a low-affinity state 4,5 . Circulating NK cells can be activated by chemokines, which causes surface LFA-1 to shift to a high-affinity state as part of 'inside-out' integrin activation 7 . Here we found that activation of LFA-1 on NK cells treated with the chemoattractant SDF-1a depended on HS1 Tyr397 independently of the total amount of LFA-1 on the cell surface. In contrast, physical 'outside-in' activation of LFA-1 by $\mathrm{Mn}^{2+}$ was unaffected. LFA-1 activation in lymphocytes requires activation of the GTPase Rap1 (refs. 8,9 ) and the function of cytohesin-1, a multidomain adaptor ${ }^{11}$. Here activation of Rap1 and the association of cytohesin-1 with LFA-1 were inhibited in cells expressing the Y397F HS1 mutant. Thus, HS1 Tyr397 seems to be 'upstream' of Rap1 and cytohesin-1 in the pathway to activating LFA-1. Rho-family GTPases are important for actin cytoskeleton dynamics 'downstream' of integrins $32,41,42$. Vav1 is important for lymphocyte activation ${ }^{43-45}$. Vav1 can interact with HS1, and this interaction depends on HS1 Tyr397 (ref. 1). Here we found that in cells expressing the Y397F HS1 mutant and responding to LFA-1 engagement, Vav1 was not activated. In those cells, Vav1 did not associate with LFA-1 or localize to the lytic synapse. In contrast, engagement of VLA- 4 by fibronectin did cause Vav1 activation in HS1-knockdown cells and cells expressing the Y397F HS1 mutant (data not shown). 
We found that HS1 Tyr378 was specifically required for NK cell chemotaxis. A series of timecourse assays designed to test the ability of NK cells to migrate toward target cells or chemoattractant showed that Tyr378 was critical for directed migration. Tyr378 became phosphorylated after the addition of chemoattractant and was required for the propagation of signaling induced by the chemoattractant. In cells expressing the Y397F HS1 mutant, initial activation of phospholipase C-b by chemoattractant was intact, but 'downstream' activation of PAK1, PI(3)K and Vav1 was impaired.

The responses of migrating leukocytes, including NK cells, to chemotactic gradients involves signaling through $\mathrm{G}$ protein-coupled receptors to Rho-family GTPases 37,38 . Rac1 activity regulates activity at the front of a migrating cell, whereas Rho functions at the back. The integration of these signaling pathways determines the directional response to chemotactic signals ${ }^{41}$. Here we found that chemoattractant stimulation activated Rac1 and Cdc42 and suppressed RhoA activity in NK cells. This activation of Rac1 and Cdc42 required HS1 Tyr378 but the downregulation of RhoA did not. Poor activation of Vav1 may have been the cause of the defective activation of Rac1 and Cdc42 induced by chemoattractant and integrin signaling. Thus, the Rho-family G proteins and Vav1 are involved 'downstream' of both Tyr378 and Tyr397 in response to chemokine and integrin stimulation, respectively.

We have established that HS1 and, more notably, phosphorylated regulation of HS1 are prominent in specific NK functions, including adhesion. We have determined that phosphorylation of HS1 Tyr397 was critical for integrin-mediated signaling required for adhesion to ICAM-1 in the context of substrate or adhesion to potential target cells.

Alternatively, phosphorylation of HS1 Tyr378 was required for chemotaxis, which is critical for reaching potential target cells. As loss of HS1 does not entirely disrupt immediate-early integrin or NK receptor signaling, we feel that HS1 is an important 'hub' molecule poised between early signaling events and actin cytoskeletal remodeling required for many distinct NK functions.

\section{METHODS}

\section{Cell culture}

Human NK cells were isolated from healthy donors with an NK cell isolation kit (Dynal) and their populations were expanded by incubation for 3-4 d with irradiated K562 cells in primary complete Iscove's medium (Iscove's modified Dulbecco's medium (Invitrogen) with 10\% ( vol/vol) human $\mathrm{AB}^{+}$serum, $2 \mathrm{mM}$ GlutaMAX (Invitrogen), $1 \mathrm{mM}$ sodium pyruvate and 100 $\mathrm{U} / \mathrm{ml}$ of recombinant interleukin 2). Primary NK cells, sorted for isolation of CD56-bright populations, were incubated for 6-9 d with irradiated K562 cells in the presence of phytohemagglutinin $(100 \mathrm{ng} / \mathrm{ml})$. Individual NK clones were isolated by sorting of CD56bright cells with a MoFlo high-speed flow cytometer (Dako Cytomation). Clones were cultured in 96-well plates with irradiated K562 cells in complete RPMI medium (described below) with $100 \mathrm{ng} / \mathrm{ml}$ of phytohemagglutinin. NK cell clones that expanded were split every $4-5 \mathrm{~d}$ at a ratio of 1:1 in complete RPMI medium (described below). Primary NK cells were used for experiments within 6 weeks of isolation. The NKL cell line (a gift from M. Robertson) and NK92 cell line (American Type Culture Collection) were grown in RPMI medium (Invitrogen) supplemented with $1 \mathrm{mM}$ sodium pyruvate, $100 \mathrm{U} / \mathrm{ml}$ of recombinant interleukin 2, 8\% (vol/ vol) FBS (Hyclone) and 8\% horse serum (Hyclone; complete RPMI medium).

\section{Knockdown and expression of HS1}

Total RNA was purified from freshly isolated primary human NK cells treated with PMA and phytohemagglutinin and was used for synthesis of first-strand cDNA with Superscript 3 (Invitrogen) and oligo(dT-14) primer (RNeasy; Qiagen); cDNA encoding human HS1 was 
amplified from the first-strand cDNA by PCR with specific primers. PCR products were cloned into BamHI and EcoRI sites of the cytomegalo-virus-based mammalian expression vector phrGFP II-C (Stratagene). This expression system fused GFP to the carboxyl terminus of HS1 and was used for all HS1 'rescue' expression. Cloned PCR products were sequenced. HS1 Tyr378 and Try397 were replaced with phenylalanine with a QuikChange kit (Stratagene). The cDNA encoding $\beta_{2}$ integrin, Pyk2 (both gifts from S. Blystone) or Vav1 (a gift from V. Tybulewicz) was subcloned into phrGFP II-C to generate carboxy-terminal GFP fusion proteins. Grb2, Lyn and WASp were amplified from cDNA derived from primary human NK cells as described above and were cloned into EcoRI and BamHI sites of phrGFP II-N (Stratagene) to generate amino-terminal GFP fusion proteins. The GFP-actin expression plasmid was provided by B. Imhof ${ }^{46}$. For knockdown of HS1, shRNA-expressing plasmids were constructed: two knockdown constructs, HS1A and HS1B, and the nontargeting control HS1sc, which was HS1A with four base-pair mismatches. For restoration of expression, shRNA-resistant HS1 expression plasmids were generated with silent mutations of three nucleotides in the target sequence. Sequences were as follows (lower case indicates bases differing from wild-type HS1): HS1A, 5'-GACTACAAGGGAGAGACGGAG-3'; HS1B, 5'CGGAGAAGTGGAGAAGCATAC-3'; HS1sc, 5'-GACTAgAAGGcAcAGAgGGAG-3'; and shRNA-resistant, 5'-

GCAGCTCTGGGATATGAtTAtAAaGGAGAGACGGAGAAA-3'. A tetracyclineinducible system (Clontech) was used for inducible knockdown of HS1. Polyclonal primary NK cells were made responsive to tetracycline by nucleofection of the ptTS-Neo plasmid (Amaxa); this plasmid contains the tetracycline-controlled transcriptional suppressor, a fusion of the tetracycline represser protein and the KRAB-AB silencing domain of the transcriptional suppressor protein Kid-1 protein. After nucleofection, NK cells were cultured for $24 \mathrm{~h}$ in complete RPMI medium with $0.75 \%$ ( $\mathrm{vol} / \mathrm{vol}$ ) DMSOandfor $48 \mathrm{~h}$ in complete RPMI medium. The aminoglycoside G418 was added at a concentration of $0.5 \mathrm{mg} / \mathrm{ml}$ and cells were maintained for $10 \mathrm{~d}$ with G418 selection in the presence of irradiated K562 cells and phytohemagglutinin $(10 \mathrm{ng} / \mathrm{ml})$. The expanded NK population was transfected with pSIREN-RetroQ-TetP plasmids (Clontech) expressing shRNA molecules targeting HS1. For knockdown, doxycycline ( $25 \mathrm{mg}$ / $\mathrm{ml}$ ) was added and HS1 expression was monitored by immunoblot every day. For restoration of HS1 expression, tetracycline-responsive HS1-knockdown cells in doxycycline for $3 \mathrm{~d}$ were transfected by nucleofection with GFP-tagged HS1 constructs. After 48 h, CD56 ${ }^{+} \mathrm{GFP}^{+}$ populations were isolated by sorting. The NK92 and NKL cell lines were made responsive to tetracycline by nucleofection of ptTS-Neo and selection in complete RPMI medium with G418 $(0.5 \mathrm{mg} / \mathrm{ml})$. Clones were assessed for tetracycline responsiveness, were transfected by nucleofection with pSIREN-RetroQ-Tetp plasmids containing shRNA constructs and were maintained in complete RPMI medium with G418 $(0.5 \mathrm{mg} / \mathrm{ml})$ and puromycin $(100 \mathrm{mg} / \mathrm{ml})$. Cells were cultured with selection for up to 4 weeks before analysis of knockdown efficiency. For HS1 'rescue' studies, the shRNA-resistant GFP-HS1 construct was transfected by nucleofection into NK92 and NKL cells and $\mathrm{GFP}^{+}$cells were isolated by sorting.

\section{Cytotoxicity assay}

For cytotoxicity assays, 96-well flat-bottomed plates (Immulon II; Dynatech) were coated overnight at $41 \mathrm{C}$ with fibronectin $(5 \mathrm{mg} / \mathrm{ml})$ in Hank's buffered saline solution. Some experiments used 96-well round-bottomed or 6-well flat-bottomed plates (Costar). K562 cells were added $\left(1 \times 10^{5}\right.$ cells per well) and were allowed to adhere for $1 \mathrm{~h}$ at $371 \mathrm{C}$ in the presence of PMA (10 ng/ml). Wells were washed with Hank's buffered saline solution. NK cells in Hank's buffered saline solution were added $\left(5 \times 10^{5}\right.$ cells per well $)$ in triplicate, followed by incubation at 37 1C. Cytotoxicity was assessed as the release of adenylate kinase into the media with a Toxilight BioAssay Kit (Cambrex). Values were normalized to the value for total release from $1 \times 10^{5} \mathrm{~K} 562$ cells with the Toxilight $100 \%$ Lysis reagent. This normalized ratio is plotted 
as the killing index. This assay has been characterized and used as a reliable alternative to standard chromium-release assays 47 .

\section{Lytic synapse and conjugate formation}

For microscopy, NKL cells stably expressing GFP-tagged proteins, at a density of $1 \times 10^{6}$ cells per ml in Hank's buffered saline solution, were pelleted with equal numbers of K562 cells, followed by incubation at $371 \mathrm{C}$. For some experiments, NK cells were incubated with Dynabeads coated with ICAM-1 and ULBP. Cells were fixed for $1 \mathrm{~h}$ at $41 \mathrm{C}$ in $3.7 \%$ (vol/vol) formaldehyde. GFP fluorescence was imaged directly. For immunofluorescence, cells were made permeable for $30 \mathrm{~s}$ in cold Hank's buffered saline solution containing $0.01 \%$ ( $\mathrm{vol} / \mathrm{vol}$ ) Nonidet P-40, then were incubated with primary and secondary antibodies. Cells were imaged with a TIRF microscope system (described below) with an APO 100× objective lens with a numerical aperture of 1.65 . For flow cytometry of cell-cell conjugates, K562 cells at a density of $1 \times 10^{6}$ cells per $\mathrm{ml}$ in Hank's buffered saline solution were stained with the red membrane dye PKH26 (Sigma), according to manufacturer's directions, and were incubated with NK cells expressing GFP-tagged proteins at a density of $1 \times 10^{6}$ cells per $\mathrm{ml}$ in RPMI medium. Cells were pelleted together and were incubated at $371 \mathrm{C}$. For untagged proteins, cells were fixed, made permeable and stained with fluorescent antibodies. The relative proportion of red, green and red-green events in each tube was determined by two-color flow cytometry with a FACSCalibur (BD Biosciences).

\section{TIRF}

Dishes with flint-glass coverslips at the bottom $(0.06-0.08 \mathrm{~mm}$ in thickness; MatTek) were coated overnight at $41 \mathrm{C}$ with ICAM-1 $(0.5 \mathrm{mg} / \mathrm{ml})$ or fibronectin $(0.5 \mathrm{mg} / \mathrm{ml})$, then were blocked with 4\% (wt/vol) casein in Hank's buffered saline solution. NK cells expressing GFPtagged proteins were allowed to adhere for various times, were fixed in $3.7 \%$ ( $\mathrm{vol} / \mathrm{vol}$ ) formaldehyde and were imaged by TIRF microscopy on an inverted Olympus IX2-UCBHamamatsu electron-multiplying charge-coupled device system with an LCPlan 20× objective lens with a numerical aperture of 0.60 for brightfield optics; a Plan Apo 60× objective lens with a numerical aperture of 1.45 for fluorescence of fields of cells; or an APO $100 \times$ objective lens with a numerical aperture of 1.65 for fluorescence of individual cells. For immunofluorescence, cells were made permeable with $0.01 \%$ (vol/vol) Nonidet P-40 and were stained with primary and secondary antibodies. Actin was visualized with Alexa Fluor 488 or Alexa Fluor 568-phalloidin (Invitrogen). For live-cell imaging, the TIRF microscope system was used. The specimen chamber was a glass-bottomed culture dish $(0.06-0.08 \mathrm{~mm}$ in thickness; MatTek). The temperature was maintained at $371 \mathrm{C}$. In cell-cell conjugate formation experiments, NKL cells stably expressing GFP alone or GFP-tagged proteins at a density of 1 $\times 10^{6}$ cells per $\mathrm{ml}$ in RPMI medium were pelleted with equal numbers of K562 cells. After 1 min, the cell pellet was dispersed onto uncoated glass-bottomed dishes and images were collected at 2- to 3-minute intervals. In adhesion experiments, glass-bottomed dishes were coated overnight at $41 \mathrm{C}$ with ICAM- $1(1 \mathrm{mg} / \mathrm{ml})$, fibronectin $(5 \mathrm{mg} / \mathrm{ml})$ or ICAM- 1 with ULBP $(0.5 \mathrm{mg} / \mathrm{ml})$ in Hank's buffered saline solution. Dishes were washed and were blocked with 3\% (wt/vol) casein in Hank's buffered saline solution. Adhesion was initiated by the addition of $15-20 \mathrm{ml}$ of a cell suspension $\left(2 \times 10^{6}\right.$ cells per $\mathrm{ml}$ in Hank's buffered saline solution). Images were acquired every $10 \mathrm{~s}$ for up to $45 \mathrm{~min}$. In chemotaxis experiments, cells were allowed to adhere to glass-bottomed dishes coated with fibronectin $(5 \mathrm{mg} / \mathrm{ml})$. A 2$\mathrm{mm}^{3}$ piece of hardened $0.5 \%$ low-melting-point agarose with SDF-1a (R\&D Systems) was placed at the periphery of the dish. 


\section{Cytokine production}

Supernatants were collected at $24 \mathrm{~h}$ from $1 \times 10^{6}$ primary NK cells cultured with PMA and Dynabeads coated with ICAM-1 and ULBP or at $24 \mathrm{~h}$ from NK cells cultured with K562 cells. Cytokines were quantified with enzyme-linked immunosorbent assay kits (R\&D Systems) according to the manufacturer's instructions.

\section{Additional methods}

Information on antibodies and reagents, immunoprecipitation and immunoblot analysis, determination of the affinity state of integrins, assays of the activity of Rho-family GTPases, and adhesion and migration assays is available in the Supplementary Methods online.

\section{Statistics}

Variance analysis followed by Student's t-test was used for statistical analysis. Results were considered significant with a $P$ value of less than 0.01 .

\section{Supplementary Material}

Refer to Web version on PubMed Central for supplementary material.

\section{Acknowledgements}

NKL cells were a gift from M. Robertson (Indiana University School of Medicine); $\beta_{2}$ integrin and Pyk2 were gifts from S. Blystone (State University of New York, Syracuse); Vav1 was a gift from V. Tybulewicz (National Institute for Medical Research, London); and the GFP-actin expression plasmid was provided by B. Imhof (University Medical Centre, Geneva). We thank the Siteman Cancer Center High Speed Sorter Core Facility, W. Eades and J. Hughes. Supported by the US National Institutes of Health (GM 38542 to J.A.C.), the National Institute of Allergy and Infectious Diseases (71429 to B.B.) and the US National Cancer Institute (P30 CA91842 to the Siteman Cancer Center High Speed Sorter Core Facility).

\section{References}

1. Gomez TS, et al. HS1 functions as an essential actin-regulatory adaptor protein at the immune synapse. Immunity 2006;24:741-752. [PubMed: 16782030]

2. Lanier LL. Natural killer cell receptor signaling. Curr Opin Immunol 2003;15:308-314. [PubMed: 12787756]

3. Allavena $\mathrm{P}$, et al. Molecules and structures involved in the adhesion of natural killer cells to vascular endothelium. J Exp Med 1991;173:439-448. [PubMed: 1671081]

4. Barber DF, Faure M, Long EO. LFA-1 contributes an early signal for NK cell cytotoxicity. J Immunol 2004;173:3653-3659. [PubMed: 15356110]

5. Donskov F, Basse PH, Hokland M. Expression and function of LFA-1 on A-NK and T-LAK cells: role in tumor target killing and migration into tumor tissue. Nat Immun 1996;15:134-146. [PubMed: 9162263]

6. Bryceson YT, March ME, Barber DF, Ljunggren HG, Long EO. Cytolytic granule polarization and degranulation controlled by different receptors in resting NK cells. J Exp Med 2005;202:1001-1012. [PubMed: 16203869]

7. Constantin $\mathrm{G}$, et al. Chemokines trigger immediate $\beta_{2}$ integrin affinity and mobility changes: differential regulation and roles in lymphocyte arrest under flow. Immunity 2000;13:759-769. [PubMed: 11163192]

8. McLeod SJ, Shum AJ, Lee RL, Takei F, Gold MR. The Rap GTPases regulate integrin-mediated adhesion, cell spreading, actin polymerization, and Pyk2 tyrosine phosphorylation in B lymphocytes. J Biol Chem 2004;279:12009-12019. [PubMed: 14701796]

9. Shimonaka M, et al. Rap1 translates chemokine signals to integrin activation, cell polarization, and motility across vascular endothelium under flow. J Cell Biol 2003;161:417-427. [PubMed: 12707305] 
10. Duchniewicz M, et al. Rap1A-deficient T and B cells show impaired integrin-mediated cell adhesion. Mol Cell Biol 2006;26:643-653. [PubMed: 16382154]

11. Kolanus W, et al. $\alpha \mathrm{L} \beta 2$ integrin/LFA-1 binding to ICAM- 1 induced by cytohesin-1, a cytoplasmic regulatory molecule. Cell 1996;86:233-242. [PubMed: 8706128]

12. Weber KS, et al. Cytohesin-1 is a dynamic regulator of distinct LFA-1 functions in leukocyte arrest and transmigration triggered by chemokines. Curr Biol 2001;11:1969-1974. [PubMed: 11747824]

13. Upshaw JL, et al. NKG2D-mediated signaling requires a DAP10-bound Grb2-Vav1 intermediate and phosphatidylinositol-3-kinase in human natural killer cells. Nat Immunol 2006;7:524-532. [PubMed: 16582911]

14. Billadeau DD, Mackie SM, Schoon RA, Leibson PJ. Specific subdomains of Vav differentially affect T cell and NK cell activation. J Immunol 2000;164:3971-3981. [PubMed: 10754287]

15. Parham P. Killer cell immunoglobulin-like receptor diversity: balancing signals in the natural killer cell response. Immunol Lett 2004;92:11-13. [PubMed: 15081521]

16. Carpen O, Virtanen I, Lehto VP, Saksela E. Polarization of NK cell cytoskeleton upon conjugation with sensitive target cells. J Immunol 1983;131:2695-2698. [PubMed: 6417230]

17. Standeven LJ, Carlin LM, Borszcz P, Davis DM, Burshtyn DN. The actin cytoskeleton controls the efficiency of killer Ig-like receptor accumulation at inhibitory NK cell immune synapses. J Immunol 2004;173:5617-5625. [PubMed: 15494512]

18. Krzewski K, Chen X, Orange JS, Strominger JL. Formation of a WIP-, WASp-, actin-, and myosin IIA-containing multiprotein complex in activated NK cells and its alteration by KIR inhibitory signaling. J Cell Biol 2006;173:121-132. [PubMed: 16606694]

19. Orange JS, et al. Wiskott-Aldrich syndrome protein is required for NK cell cytotoxicity and colocalizes with actin to NK cell-activating immunologic synapses. Proc Natl Acad Sci USA 2002;99:11351-11356. [PubMed: 12177428]

20. Wu H, Parsons JT. Cortactin, an 80/85-kilodalton pp60src substrate, is a filamentous actin binding protein enriched at the cell cortex. J Cell Biol 1993;120:1417-1426. [PubMed: 7680654]

21. Weaver AM, et al. Cortactin promotes and stabilizes Arp2/3-induced actin filament network formation. Curr Biol 2001;11:370-374. [PubMed: 11267876]

22. Kitamura D, Kaneko H, Miyagoe Y, Ariyasu T, Watanabe T. Isolation and characterization of a novel human gene expressed specifically in cells of hematopoietic lineage. Nucleic Acids Res 1989;17:9367-9379. [PubMed: 2587259]

23. Uruno T, Zhang P, Liu J, Hao JJ, Zhan X. Haematopoietic lineage cell-specific protein 1 (HS1) promotes actin-related protein (Arp) 2/3 complex-mediated actin polymerization. Biochem $\mathrm{J}$ 2003;371:485-493. [PubMed: 12534372]

24. Brunati AM, et al. Thrombin-induced tyrosine phosphorylation of HS1 in human platelets is sequentially catalyzed by Syk and Lyn tyrosine kinases and associated with the cellular migration of the protein. J Biol Chem 2005;280:21029-21035. [PubMed: 15795233]

25. Ruzzene M, Brunati AM, Marin O, Donella-Deana A, Pinna LA. SH2 domains mediate the sequential phosphorylation of HS1 protein by p72syk and Src-related protein tyrosine kinases. Biochemistry 1996;35:5327-5332. [PubMed: 8611520]

26. Yamanashi Y, et al. Identification of HS1 protein as a major substrate of protein-tyrosine kinase(s) upon B-cell antigen receptor-mediated signaling. Proc Natl Acad Sci USA 1993;90:3631-3635. [PubMed: 7682714]

27. Taniuchi I, et al. Antigen-receptor induced clonal expansion and deletion of lymphocytes are impaired in mice lacking HS1 protein, a substrate of the antigen-receptor-coupled tyrosine kinases. EMBO J 1995;14:3664-3678. [PubMed: 7641686]

28. Kahner BN, et al. Hematopoietic lineage cell specific protein 1 (HS1) is a functionally important signaling molecule in platelet activation. Blood 2007;110:2449-2456. [PubMed: 17579181]

29. Cairo CW, Mirchev R, Golan DE. Cytoskeletal regulation couples LFA-1 con-formational changes to receptor lateral mobility and clustering. Immunity 2006;25:297-308. [PubMed: 16901728]

30. Green CE, et al. Dynamic shifts in LFA-1 affinity regulate neutrophil rolling, arrest, and transmigration on inflamed endothelium. Blood 2006;107:2101-2111. [PubMed: 16269618]

31. Loubani O, Hoskin DW. Paclitaxel inhibits natural killer cell binding to target cells by downregulating adhesion molecule expression. Anticancer Res 2005;25:735-741. [PubMed: 15868904] 
32. Burridge K, Wennerberg K. Rho and Rac take center stage. Cell 2004;116:167-179. [PubMed: 14744429]

33. Djeu JY, Jiang K, Wei S. A view to a kill: signals triggering cytotoxicity. Clin Cancer Res 2002;8:636640. [PubMed: 11895890]

34. Woolf E, et al. Lymph node chemokines promote sustained T lymphocyte motility without triggering stable integrin adhesiveness in the absence of shear forces. Nat Immunol 2007;8:1076-1085. [PubMed: 17721537]

35. Li Z, et al. Roles of PLC- $\beta 2$ and $-\beta 3$ and PI3Kg in chemoattractant-mediated signal transduction. Science 2000;287:1046-1049. [PubMed: 10669417]

36. Phee H, Abraham RT, Weiss A. Dynamic recruitment of PAK1 to the immunological synapse is mediated by PIX independently of SLP-76 and Vav1. Nat Immunol 2005;6:608-617. [PubMed: 15864311]

37. Maghazachi AA. Role of the heterotrimeric G proteins in stromal-derived factor-1a-induced natural killer cell chemotaxis and calcium mobilization. Biochem Biophys Res Commun 1997;236:270-274. [PubMed: 9240423]

38. Haddad E, et al. The interaction between Cdc42 and WASP is required for SDF-1-induced Tlymphocyte chemotaxis. Blood 2001;97:33-38. [PubMed: 11133739]

39. Tehrani S, Tomasevic N, Weed S, Sakowicz R, Cooper JA. Src phosphorylation of cortactin enhances actin assembly. Proc Natl Acad Sci USA 2007;104:11933-11938. [PubMed: 17606906]

40. Luo C, et al. CXCL12 induces tyrosine phosphorylation of cortactin, which plays a role in CXC chemokine receptor 4-mediated extracellular signal-regulated kinase activation and chemotaxis. $\mathbf{J}$ Biol Chem 2006;281:30081-30093. [PubMed: 16905744]

41. Hall A. Rho GTPases and the control of cell behaviour. Biochem Soc Trans 2005;33:891-895. [PubMed: 16246005]

42. Khurana D, Leibson PJ. Regulation of lymphocyte-mediated killing by GTP-binding proteins. J Leukoc Biol 2003;73:333-338. [PubMed: 12629146]

43. Ardouin L, et al. Vav1 transduces TCR signals required for LFA-1 function and cell polarization at the immunological synapse. Eur J Immunol 2003;33:790-797. [PubMed: 12616499]

44. Billadeau DD, et al. The Vav-Rac1 pathway in cytotoxic lymphocytes regulates the generation of cell-mediated killing. J Exp Med 1998;188:549-559. [PubMed: 9687532]

45. Galandrini R, Palmieri G, Piccoli M, Frati L, Santoni A. Role for the Rac1 exchange factor Vav in the signaling pathways leading to NK cell cytotoxicity. J Immunol 1999;162:3148-3152. [PubMed: 10092764]

46. Ballestrem C, Wehrle-Haller B, Imhof BA. Actin dynamics in living mammalian cells. J Cell Sci 1998;111:1649-1658. [PubMed: 9601095]

47. Radaeva S, Sun r, Jaruga B, Nguyen V, Tian Z, Gao B. Natural killer cells ameliorate liver fibrosis by killing activated stellate cells in NKG2D-dependent and tumor necrosis factor-related apoptosisinducing ligand dependent manners. Gastroenterology 2006;130:435-452. [PubMed: 16472598] 
a
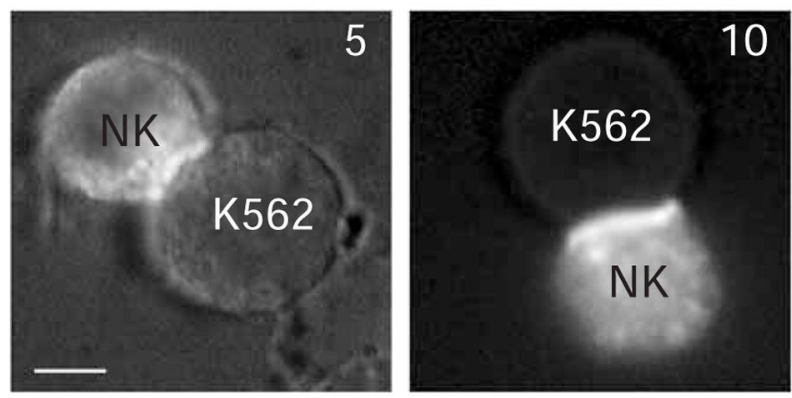

Time (min)

IP: HS1

IB: $p$-Tyr

IP: HS1

IB: HS1

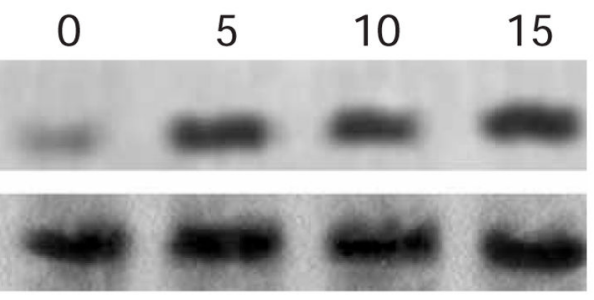

b
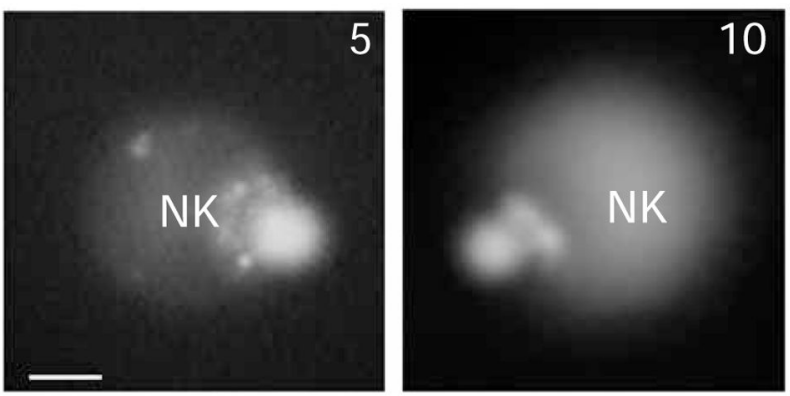

Time (min)

IP: HS1

IB: p-Tyr

IP: HS1

IB: HS1
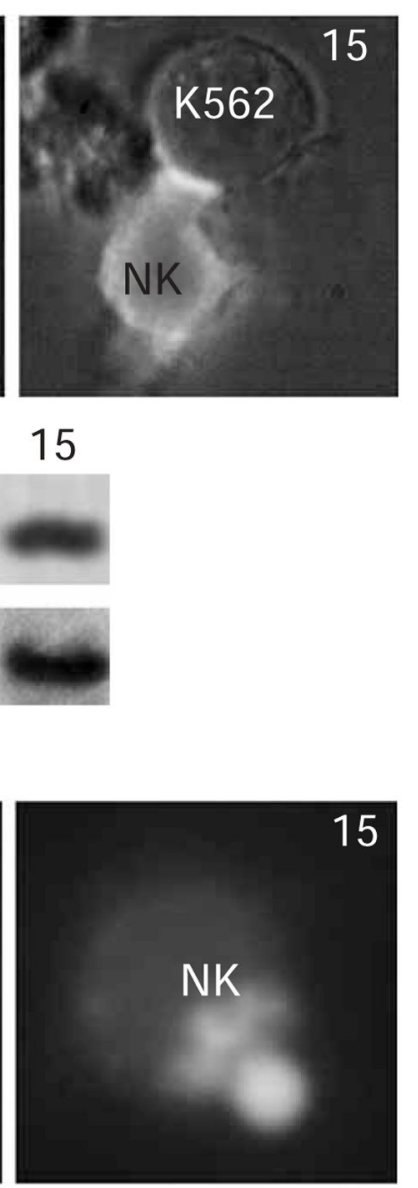

5

15

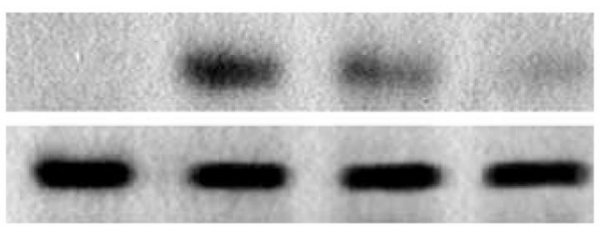

Figure 1.

Localization and phosphorylation of HS1 during the formation of lytic synapses. Top rows, localization of primary NK cells expressing GFP-HS1 incubated for 5, 10 or 15 min (top right corners) at 37 1C with K562 target cells (a) or Dynabeads coated with ICAM-1 and ULBP (b). Scale bars, $3 \mathrm{~mm}$. Below, immunoprecipitation (IP) of HS1 from cell lysates followed by immunoblot analysis (IB) with monoclonal antibody 4G10 to assess phosphorylated tyrosine (p-Tyr) or with antibody to HS1 (anti-HS1) to show equal loading of lanes. For all immunoblot analyses in all figures, an equal amount of protein was loaded into each well, as determined by the Bradford assay and Ponceau staining. Data are representative of three separate experiments (images) or at least three separate experiments (immunoblots). 
a

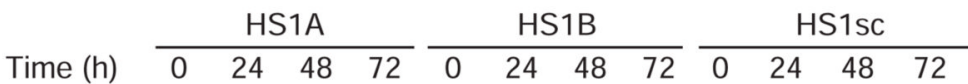

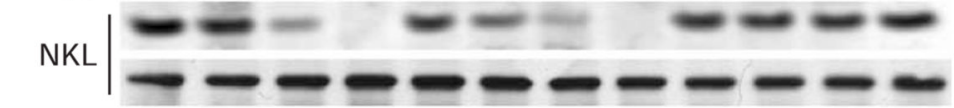

IP: HS1

IB: HS1

Primary| $\mid---\infty-\infty--\infty-\infty=$

WCL IB: actin

IP: HS1

IB: HS1

b

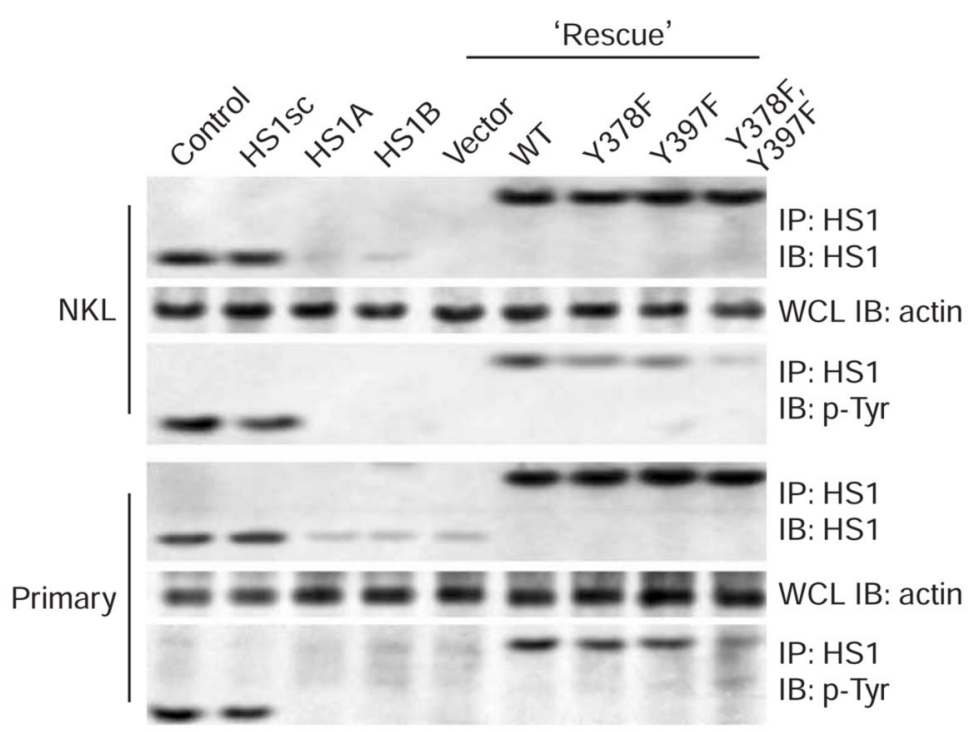

C

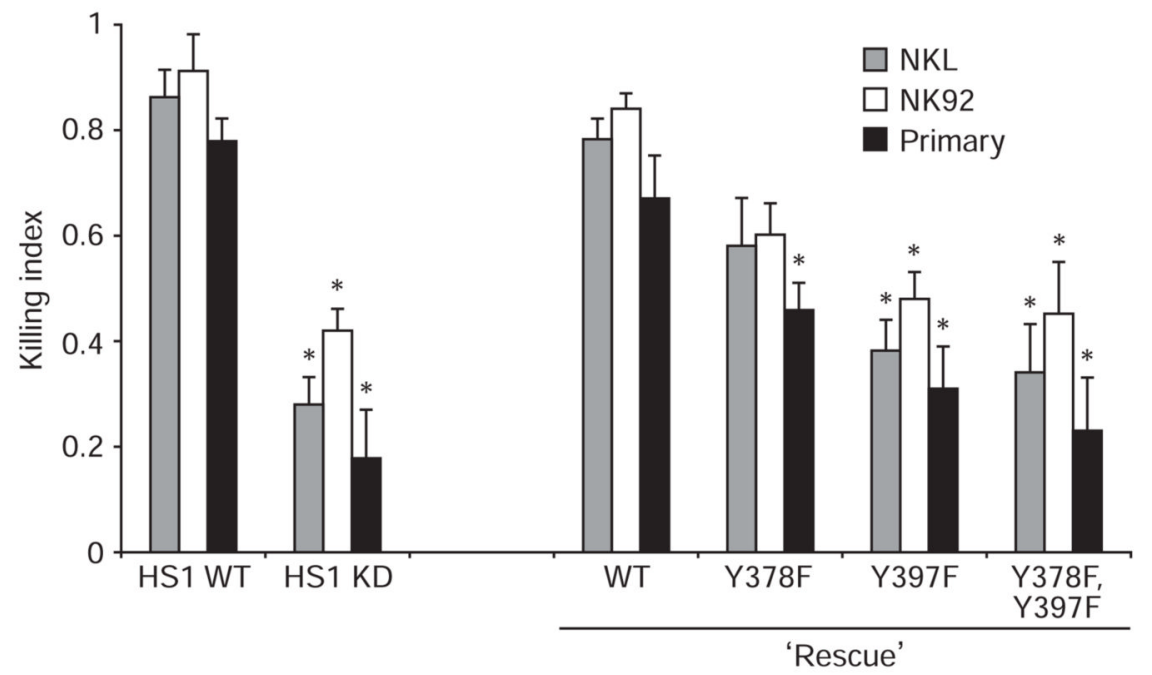

Figure 2.

Function of HS1 in cytolysis. (a) HS1 knockdown in NKL cells and primary NK cells expressing a tetracycline-inducible shRNA sequence targeting HS1 (HS1A or HS1 B) or a nontargeting control shRNA (HS1sc) and treated for 0-72 h (above lanes) with doxycycline; HS1 was immunoprecipitated and analyzed by immunoblot with anti-HS1. (b) Restoration of HS1 expression in HS1-knockdown cells treated with doxycycline and then transfected by nucleofection $72 \mathrm{~h}$ later with shRNA-resistant GFP-tagged wild-type or mutant HS1 constructs (above lanes), followed by immunoprecipitation of HS1 at $72 \mathrm{~h}$ later. For immunoblot analysis of phosphorylated tyrosine, cells were treated with $2 \mathrm{mM} \mathrm{Na}_{3} \mathrm{VO}_{4}$ for $15 \mathrm{~min}$ before lysis. HS1 was immunoprecipitated and analyzed by immunoblot with anti-HS1. WCL $(\mathbf{a}, \mathbf{b})$, immunoblot 
of one tenth of the preimmunoprecipitation whole-cell lysate with anti-actin to show equal loading of lanes, (c) Cytolysis assays of NK cell populations and K562 target cells combined for $4 \mathrm{~h}$ at $37 \mathrm{CC}$, presented as the killing index, based on the release of adenylate kinase into the media. WT, wild-type; KD, knockdown. ${ }^{*}, P<0.01$. Data are representative of at least three separate experiments (mean and s.e.m. of triplicate wells, $\mathbf{c}$ ). 
a

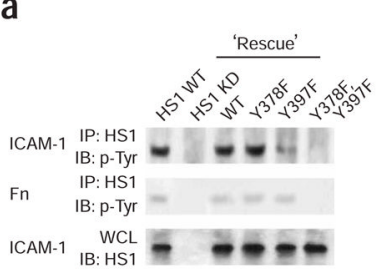

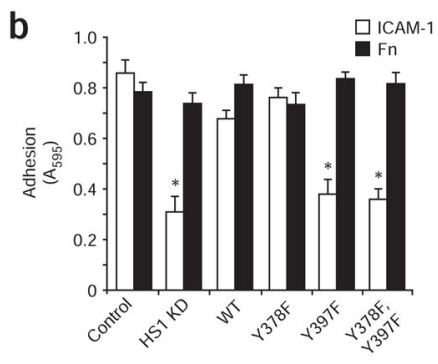

d
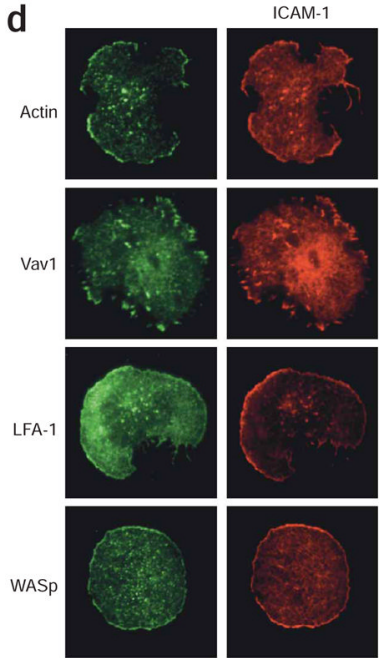

c
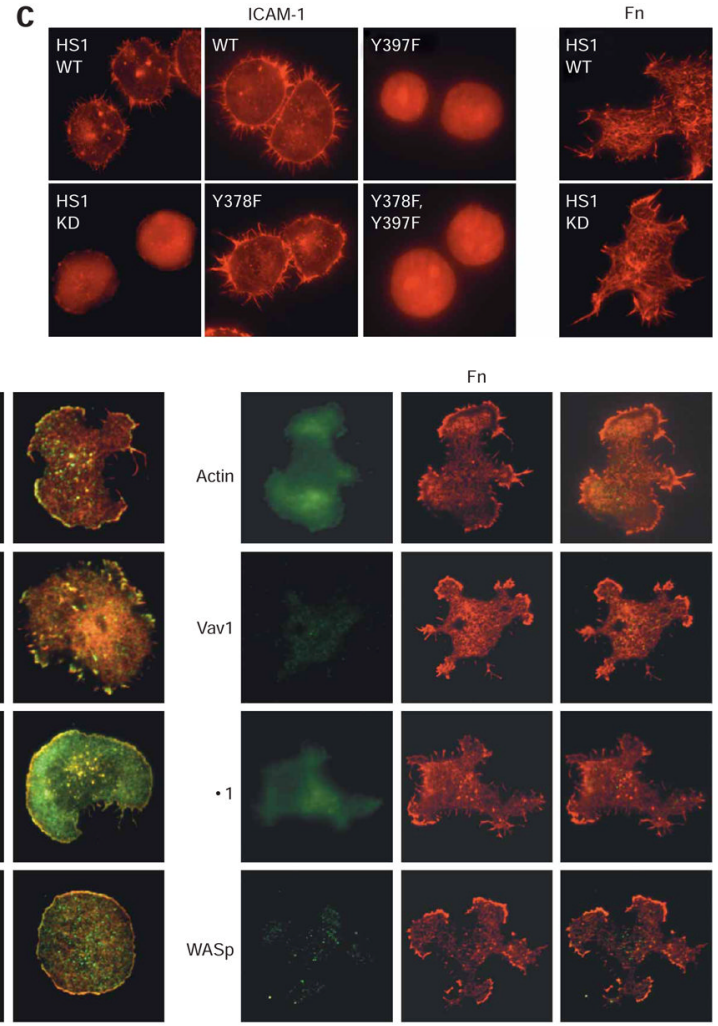

Figure 3.

HS1 in NK cell adhesion.

(a) Tyrosine phosphorylation of HS1 during the adhesion of primary NK cells expressing shRNA and/or 'rescue' constructs in six-well plates coated with ICAM-1 or fibronectin (Fn) in the presence of PMA (10 ng/ml); after $15 \mathrm{~min}$, HS1 was immunoprecipitated and analyzed by immunoblot with antibody to phosphorylated tyrosine. WCL, immunoblot analysis of one tenth of the preimmunoprecipitation whole-cell lysate of NK cells on ICAM-1 with anti-HS1 to assess total HS1.

(b) Adhesion of primary NK cell populations to 96-well plates coated with ICAM-1 or fibronectin, in the presence of PMA, assessed as absorbance at $595 \mathrm{~nm}\left(A_{595}\right)$.

$*, P<0.01$. (c) Morphology of primary NK cells (as described in a) allowed to adhere to ICAM-1 or fibronectin-coated six-well plates with glass coverslips on the bottom, then fixed and stained with Alexa Fluor 568-phalloidin. (d) TIRF microscopy of HS1 localization in NKL cells expressing GFP-HS1, allowed to adhere to ICAM-1 - or fibronectin-coated plates, then fixed, made permeable and stained with antibodies specific for various proteins (left margins). Original magnification, $\times 100(\mathbf{c}, \mathbf{d})$. Data are representative of at least three separate experiments (mean and s.e.m. of triplicate wells, b). 
a

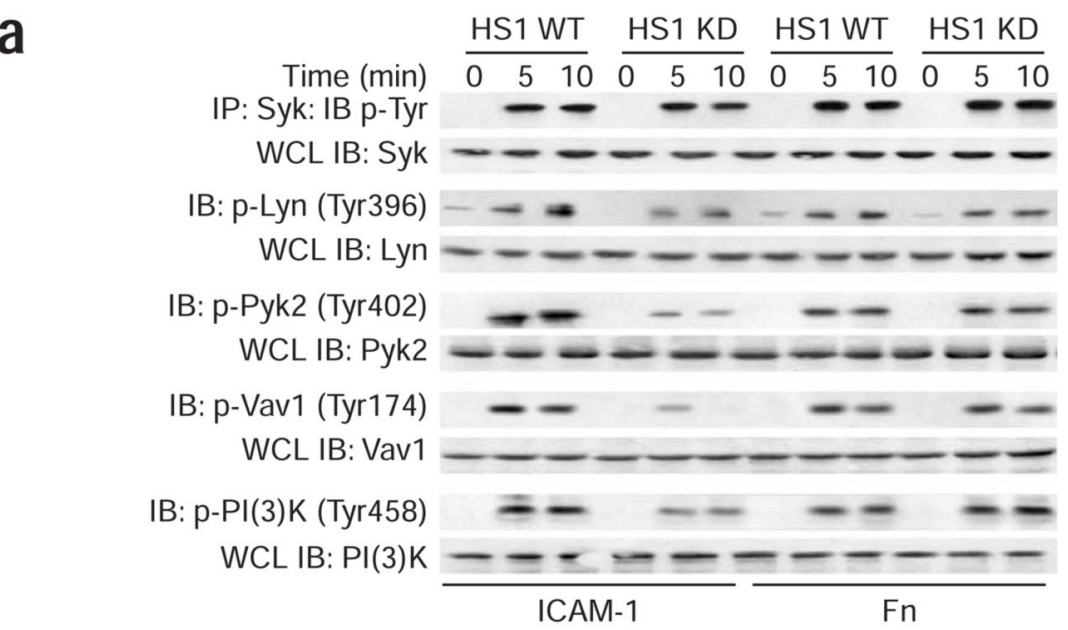

b

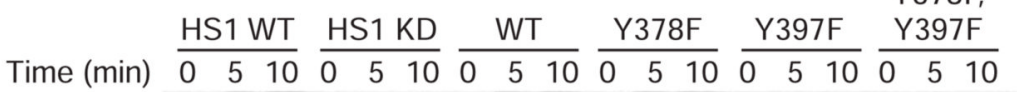

IP: ${ }_{2}$ IB: p-Vav1 (Tyr174)

IP: $\bullet 2$ IB: Vav1

$---$

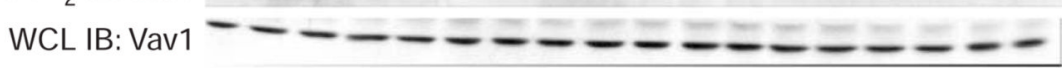

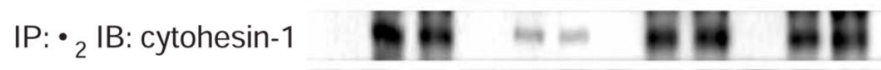

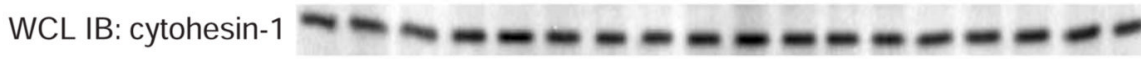

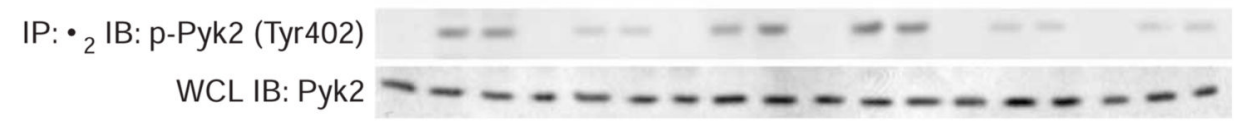

C

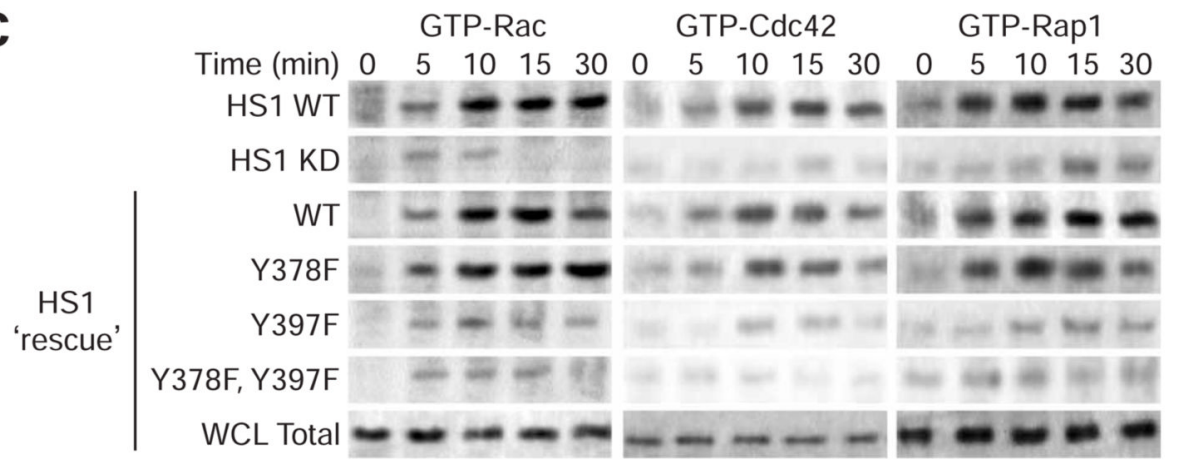

Figure 4.

Integrin-mediated signaling in HS1-deficient NK cells and NK cells expressing HS1 mutants, (a) HS1 in the activation of specific signaling proteins 'downstream' of LFA-1 in control primary NK cells or primary NK cells expressing shRNA targeting HS1; cells were allowed to adhere to ICAM-1 or fibronectin in the presence of PMA for 0,5 or 10 min at $371 \mathrm{C}$, then were analyzed by immunoblot of lysates with antibodies specific for phosphorylated molecules (phosphorylated residues in parentheses), except Syk, which was immunoprecipitated and then analyzed with antibody to phosphorylated tyrosine. (b) Association of specific signaling proteins with integrin $\beta_{2}$ in primary NK cells allowed to adhere to ICAM-1 in the presence of PMA for 0,5 or 10 min at 37 1C; lysates immunoprecipitated with Dynabeads coated with 
anti- $\beta_{2}$ integrin were analyzed by immunoblot with monoclonal antibodies specific for the proteins along the left margin. WCL $(a, b)$, immunoblot of one tenth of the preimmunoprecipitation whole-cell lysate. (c) Immunoblot analysis of GTP-bound Rac, Cdc42 and Rap1 precipitated from lysates of primary NK cells allowed to adhere to ICAM-1 in the presence of PMA for 0, 5 or $10 \mathrm{~min}$ at $37 \mathrm{CC}$. WCL, immunoblot analysis of one tenth of the preimmunoprecipitation whole-cell lysates of HS1 -knockdown cells to show that the effects produced were not due to loss of protein expression (such as Rac, Cdc42 or Rapl) with loss of HS1 expression. Data are representative of at least three separate experiments. 

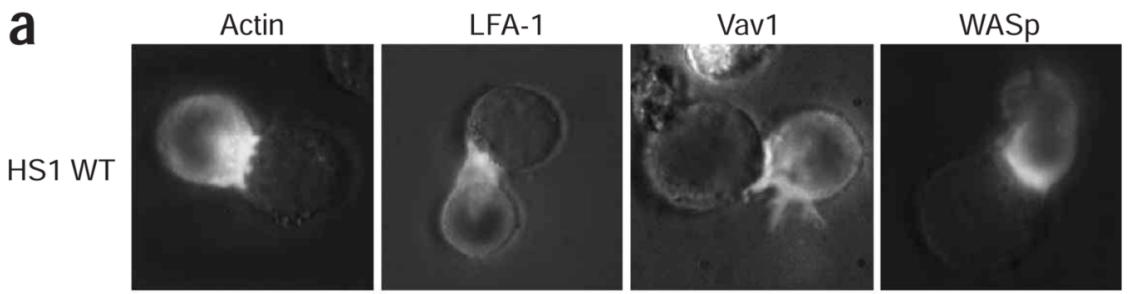

HS1 KD
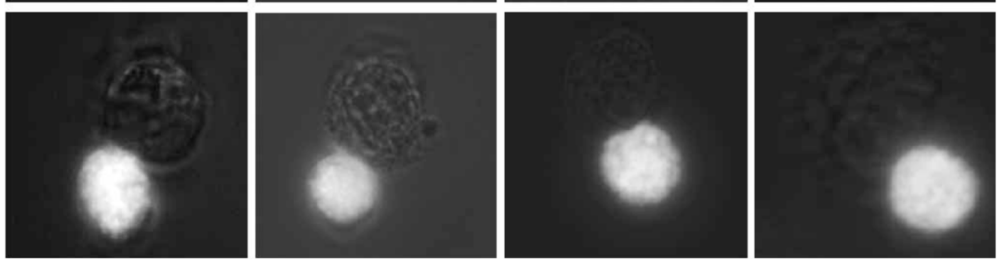

b

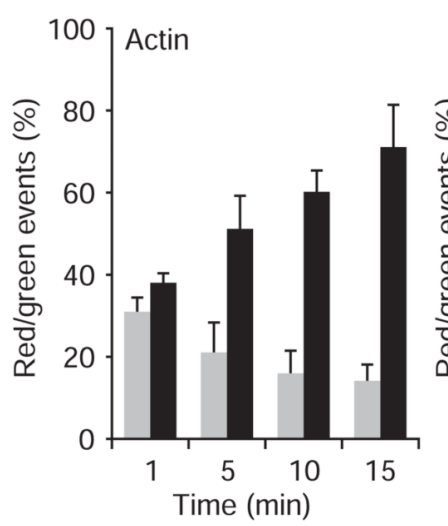

100
¿ $80-$ Vav1

HS1 WT

HS1 KD

C

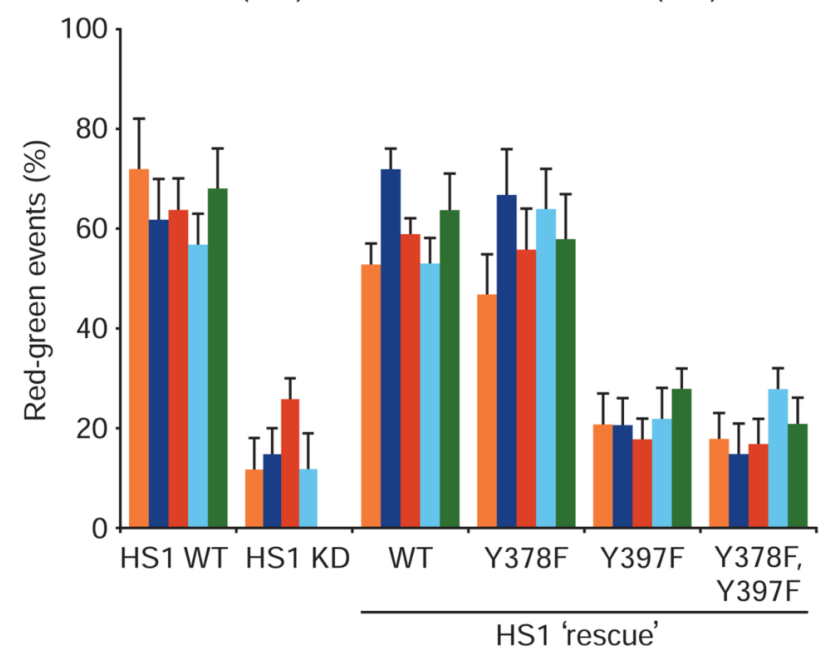

Figure 5.

Function of HS1 in the formation of NK cell-target cell lytic synapses and conjugates, (a) Overlays of fluorescence and transmitted-light images of fixed control (HS1 WT) or HS1 knockdown NKL cells stably expressing GFP-tagged proteins and incubated for $15 \mathrm{~min}$ at 37 $1 \mathrm{C}$ with target K562 cells. Original magnification, $\times 100$. (b) Two-color flow cytometry of the time course of cell-cell conjugate formation by K562 cells stained with the red dye PKH26 and incubated for 1-15 min (horizontal axes) at $371 \mathrm{C}$ with control or HS1-knockdown NKL cells expressing GFP-tagged actin or Vav1, presented as the percentage of red-green events relative to total events, (c) Quantification of cell-cell conjugate formation by K562 cells labeled with PKH26 and incubated for $15 \mathrm{~min}$ at 37 1C with NKL cells (horizontal axis) expressing 
various GFP-tagged proteins (key), presented as described in b. Data are representative of at least three separate experiments (error bars, s.e.m.). 


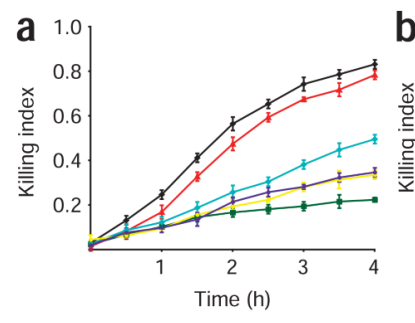

- WT Control - KD: WT 'rescue'

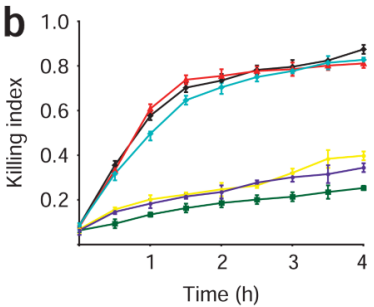

KD: Y378F, Y397F 'rescue' - KD: Y378F 'rescue'

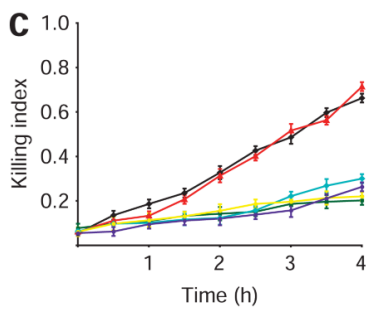

KD: Y397F 'rescue'

Figure 6.

Selective functions for HS1 tyrosine residues in NK cell migration and cytolysis. Killing index of primary NK cell populations expressing shRNA and/or 'rescue' constructs (key) and incubated for $4 \mathrm{~h}$ at $37 \mathrm{1C}$ with K562 target cells in 96-well flat-bottomed plates (a), 96-well round-bottomed plates (b) or 6-well flat-bottomed plates (c), assessed on the basis of release of adenylate kinase into the media. Data are the mean \pm s.e.m. of triplicate wells from at least three separate experiments. 


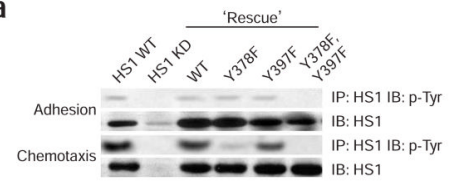

b

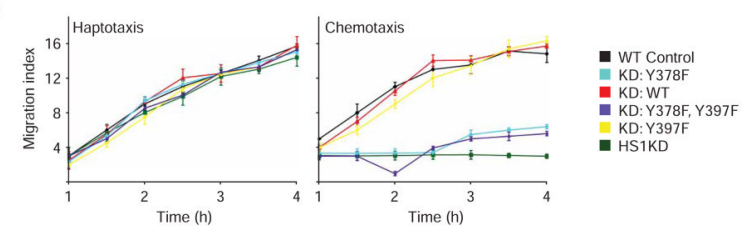

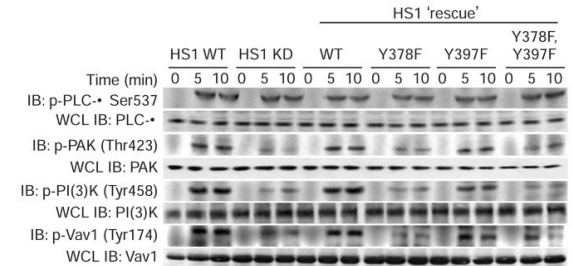

d

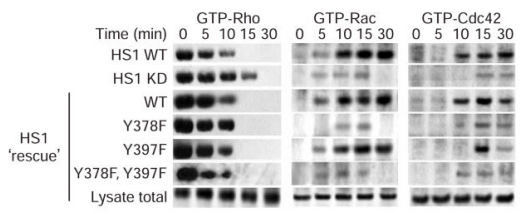

Figure 7.

Function of HS1 in chemotaxis. (a) Tyrosine phosphorylation of HS1 in primary NK cells allowed to adhere to fibronectin-coated six-well plates for $15 \mathrm{~min}$ at $37 \mathrm{CC}$ in the presence of PMA (Adhesion), or treated as described above and then exposed for 15 min to SDF-1a (Chemotaxis), followed by immunoprecipitation of HS1 and immunoblot analysis with antibody to phosphorylated tyrosine. Bottom blot (IB: HS1), one tenth of the preimmunoprecipitation whole-cell lysate. (b) Transwell assay of the migration of primary NK cells (Supplementary Methods), (c) Activity of signaling proteins in primary NK cells adhering to fibronectin and treated with SDF-1a (as described in a), analyzed by immunoblot with monoclonal antibodies specific for the proteins along the left margin. WCL, immunoblot analysis of one tenth of the preimmunoprecipitation whole-cell lysate. PLC-b, phospholipase C-b. (d) Signaling by Rho-family GTPases in NKL cell populations treated as described in a; lysates were 'CRIB precipitated' on GST-PBD agarose (PAK-binding domain) or GST-RBD agarose (Rhotekin-binding domain) and analyzed by immunoblot. Lysate total, immunoblot analysis of one tenth of the preimmunoprecipitation whole-cell lysate of HS1-knockdown cells. Data are representative of at least three separate experiments (mean \pm s.e.m. of triplicate wells, b). 
a
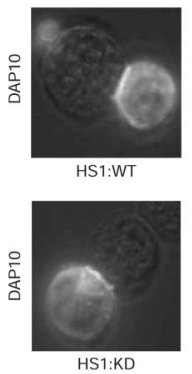

b

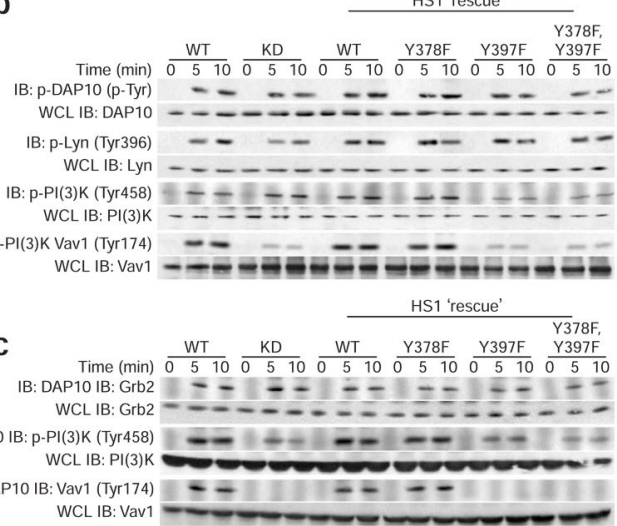

d

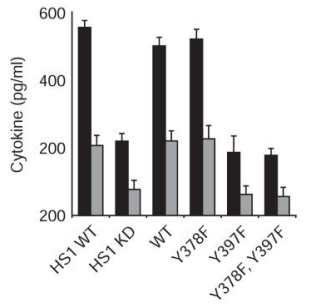

e GTP-Rac GTP-Cdc42 GTP-Rap1

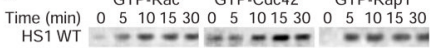
HS1 KD

$\frac{\text { 'Rescue' }}{W T}$

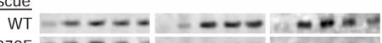

Y $378 \mathrm{~F}=--0-0-1-0=0$

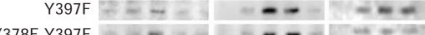
WCL: Total $\mathbf{0}=\mathbf{0}=0$

Figure 8.

Function of HS1 in NK receptor signaling, (a) DAP10 localization in control and HS1knockdown primary NK cells and target K562 cells pelleted together, incubated for 10 min at $371 \mathrm{C}$, then fixed and stained with anti-DAP10. Original magnification, $\times 100$. (b) Activation of signaling proteins in primary NK cells expressing shRNA and/or 'rescue' constructs and incubated for 0,5 or 10 min at $371 \mathrm{C}$ with Dynabeads coated with ICAM-1 and ULBP. For DAP10, lysates were immunoprecipitated with anti-DAP10 and analyzed by immunoblot with antibody to phosphorylated tyrosine; for Lyn, Vav1 and PI(3)K, immunoblots of lysates were analyzed with antibodies specific for phosphorylated molecules, (c) Association of proteins with DAP10 in primary NK cells incubated with Dynabeads; lysates immunoprecipitated with anti-DAP10 were analyzed by immunoblot with monoclonal antibodies specific for proteins along left margin. WCL (b,c), immunoblot analysis of one tenth of the preimmunoprecipitation whole-cell lysate. (d) Concentration of the cytokines interferon-g (black) and tumor necrosis factor (gray) in the media of primary NK cell populations cultured with K562 cells, (e). Rhofamily GTPase activity in NKL cell populations incubated with Dynabeads as describe in $\mathbf{b}$, assessed by analysis of lysates for GTP-bound Rac, Cdc42 and Rap1. WCL, immunoblot analysis of one tenth of the preimmunoprecipitation whole-cell lysate from HS1 -knockdown cells. Data are representative of at least three separate experiments (mean and s.e.m. of triplicate wells, d). 
Table 1

High-affinity state of integrins expressed on primary NK or NKL cells

\begin{tabular}{|c|c|c|c|c|}
\hline \multirow[b]{2}{*}{ Primary NK cells } & \multicolumn{2}{|c|}{$\beta_{2}$ integrin } & \multicolumn{2}{|c|}{$\beta_{1}$ integrin } \\
\hline & Control & SDF-1 $\alpha$ & Control & SDF-1 $1 \alpha$ \\
\hline $\begin{array}{l}\text { Wild-type control } \\
\text { HS1 knockdown } \\
\text { HS1 'rescue' } \\
\text { Wild-type } \\
\text { Y378F } \\
\text { Y397F } \\
\text { Y378F, Y397F }\end{array}$ & $\begin{array}{c}8.3 \pm 2.1 \\
5.4 \pm 2.6 \\
10.1 \pm 2.7 \\
6.5 \pm 1.8 \\
7.2 \pm 2.5 \\
5.7 \pm 1.4\end{array}$ & $\begin{array}{l}38.3 \pm 6.7 \\
14.6 \pm 4.8 \\
42.6 \pm 5.4 \\
26.9 \pm 7.5 \\
18.3 \pm 4.3 \\
16.8 \pm 3.7\end{array}$ & $\begin{array}{l}6.3 \pm 2.3 \\
4.3 \pm 1.4 \\
5.7 \pm 1.9 \\
6.4 \pm 2.7 \\
7.9 \pm 3.6 \\
5.1 \pm 1.8\end{array}$ & $\begin{array}{l}42.5 \pm 2.1 \\
28.7 \pm 3.5 \\
36.4 \pm 7.3 \\
42.6 \pm 5.3 \\
40.2 \pm 7.8 \\
50.0 \pm 5.9\end{array}$ \\
\hline NKL cells & Control & $\mathrm{Mn}^{2+}$ & & \\
\hline $\begin{array}{l}\text { Wild-type control } \\
\text { HS1 knockdown } \\
\text { HS1 'rescue' } \\
\text { Wild-type } \\
\text { Y378F } \\
\text { Y397F } \\
\text { Y378F, Y397F }\end{array}$ & $\begin{array}{l}8.4 \pm 1.7 \\
7.1 \pm 2.3 \\
5.7 \pm 2.1 \\
4.8 \pm 1.9\end{array}$ & $\begin{array}{l}32.4 \pm 5.3 \\
24.3 \pm 5.1 \\
28.5 \pm 3.9 \\
29.3 \pm 4.2 \\
31.5 \pm 3.6 \\
22.8 \pm 5.4\end{array}$ & & \\
\hline
\end{tabular}

Flow cytometry of primary NK cells incubated with activation-specific antibodies to LFA-1 (mAb24) or integrin $\beta 1$ (9EG7). Data are the mean channel fluorescence \pm s.e.m. of at least three separate experiments. 